\title{
Application of Wireless Power Transfer for Home Appliances using Inductive Resonance Coupling
}

\author{
Arjun Sharma \\ Senior Lecturer, Department of Mechatronics \\ Advanced Technical Training Centre (ATTC),Bardang, Sikkim, India
}

\begin{abstract}
Now a day the need of wireless technology is increased because of mobility, low cost and easy to maintain. This technology is commonly used for transferring the digital information over the air medium. The wireless power transmission technology is well suited for transferring power to the home appliances without wires such as smart phones, tablets, LED TV's, DVD player and home lighting systems and also for military surveillances. Commonly the Electro Magnetic (EM) waves are used for transferring the electric power through air to get a device powered. This paper proposes the Resonant coupling technique to transfer high electrical power through air medium.
\end{abstract}

Keywords - Inductance, Resonant, Wireless power transmission, Energy Coupling.

\section{I.INTRODUCTION}

Commonly the electrical energy is transferred to the load through the wire it may be copper, aluminum or any conductive material. But there are more possibilities to transfer the electrical energy through wireless medium by using various technologies, depends on the range of transmission the technology will change. The first wireless electrical power transmission technology was developed on 1890's by Nikola Tesla by implementing the wardenclyffe tower; it works around $40 \mathrm{Km}$ range. Generally 20 to 30 percent of electrical energy is loss while transferring from one source to destination, to avoid the loss and acquiring the $100 \%$ electric power to the destination we need a wireless electric power transmission technology. The need of wireless electric power transmission is for increasing the power demand, transferring electric power to the every corner of this world, for efficient and reliable power transmission and reduces the cost and maintenance of wired power transmission. The rest part of this paper describes about wireless power transfer techniques, microwave power transmission and inductive resonant coupling method.

\section{WIRELESS ELECTRIC POWER TRANSFER TECHNOLOGY}

The wireless electric power transmission is a method of conveying the electric power from source to destination (load). This type of power transmission is more efficient and low cost of transmission without installing huge amount of wires. There are three approaches are used to transfer the electricity through wireless medium such are Short range, Mid range and long range. 


\section{A. Short Range}

The transformer is the good example of short range wireless electric power transmission which works based on inductive energy coupling methodology. The transformer transfers the electrical energy as an Electromagnetic field.

Fig.1Typical construction of Transformer

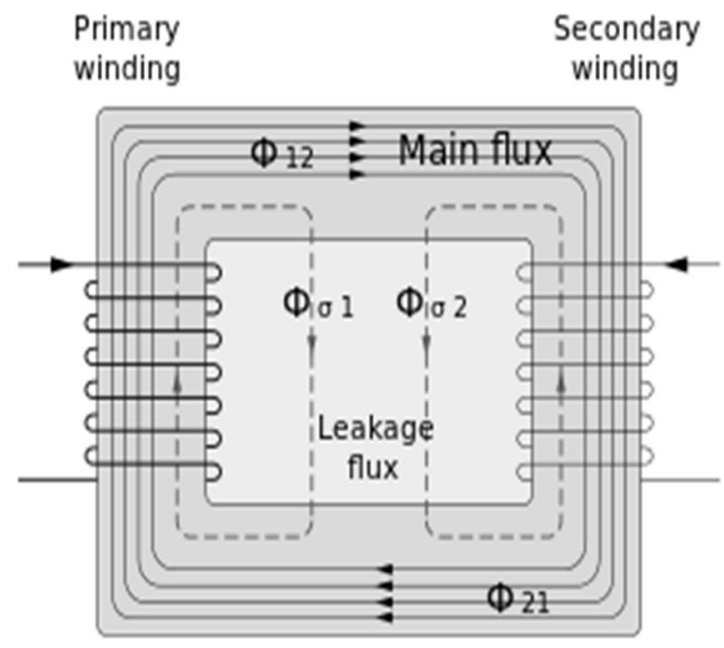

When the electric current pass through the coil then there is some magnetic field will creates inside and outside the coil. The magnetic field creates inside the coil is called strong field and the magnetic field creates around the coil is called weak field. Two coils are used for transferring the electric power on is primary coil which is directly connected to the power source and another one is secondary coil which receives the electricity by means of magnetic field and it is connected to the load or any other circuit. The Electro Magnetic Flux (EMF) is created between the primary and secondary coil, the secondary coil is inducted by the magnetic field of primary coil.

\section{B. Long Range}

For transferring the electric power through the long distance some approaches are used such as microwave power transmission, laser power transmission and solar power satellite. For the long distance electric power transfer, line of sight is required. Mostly the microwave power transfer technology is used for transferring the electric power from source to destination of long distance. First the electrical energy is converted into microwave energy and it is transferred through the antenna and then the microwave signal is converted into electrical energy at the destination side. For converting the Electrical Power in to the microwave signal we need to convert the $\mathrm{AC}$ signal in to the $\mathrm{DC}$ signal then the DC signal is converted into AC signal at receiver side.

Fig.2 Construction of Microwave power transmission

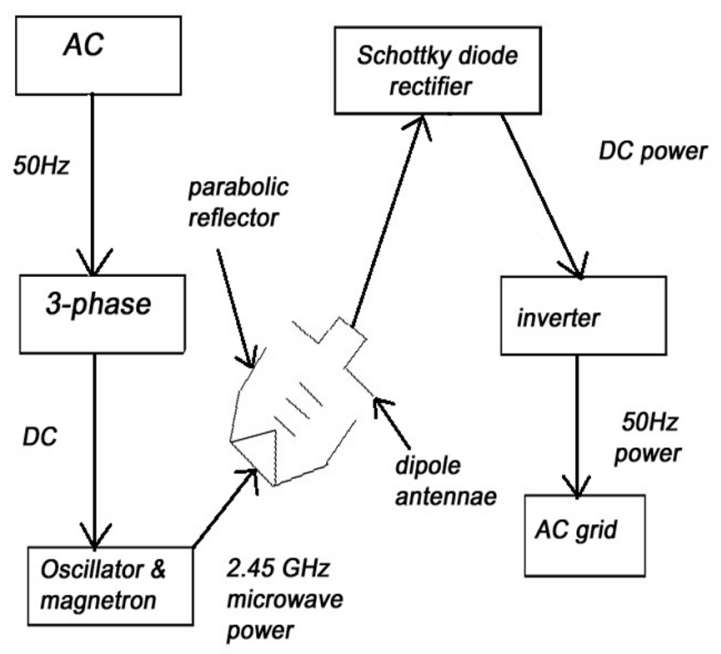




\section{Mid Range Transmission}

For the mid range of wireless power transfer generally three approaches are used such as inductive coupling, resonant inductive coupling and air ionization. The combination of resonant and inductance which is called inductive resonant that can be used to transfer electric power on some range of distance safely and effectively. Resonant are used to make the strong interaction between the primary and secondary coil and the inductance is used to induce the magnetic flux between the two coils. When two objects operate at same frequency then it can exchange energy between them without any noise. The mid range power transmission is well suited for home based wireless power transmission. With the help of near field inductance coupling we can power the home appliances.

\section{INDUCTIVE RESONANT COUPLING}

The resonant circuit is used for increasing the inductive power of electric power transmission. The resonant inductive coupling method consist of two set of coils primary coil and secondary coil each having a equivalent turn and the primary coil is connected to the power source, the current flows through the coil will induce the magnetic flux then the magnetic flux attracted by the secondary coil which is called tunneling and the secondary coil is connected to the load. When two objects having same frequency then they can transfer the electrical energy among them. To create a resonant two capacitors are need to connect to the each end of the secondary coil which is connected to the load. Some of the advantages of resonant inductive coupling are as follows

- Resonant inductive coupling is highly efficient

- It is directional coupling

- It can traverse longer distance than inductive coupling

- It is very portable

- More number of devices can use a single source

- It can be used for charging mobile devices and electric vehicles.

- Easy to maintenance

- Low cost

- Line of sight is no necessary

- Need for battery is eliminated

\section{OPERATION OF INDUCTIVE RESONANT COUPLING}

Fig.3 Block diagram of Resonant Inductive Coupling

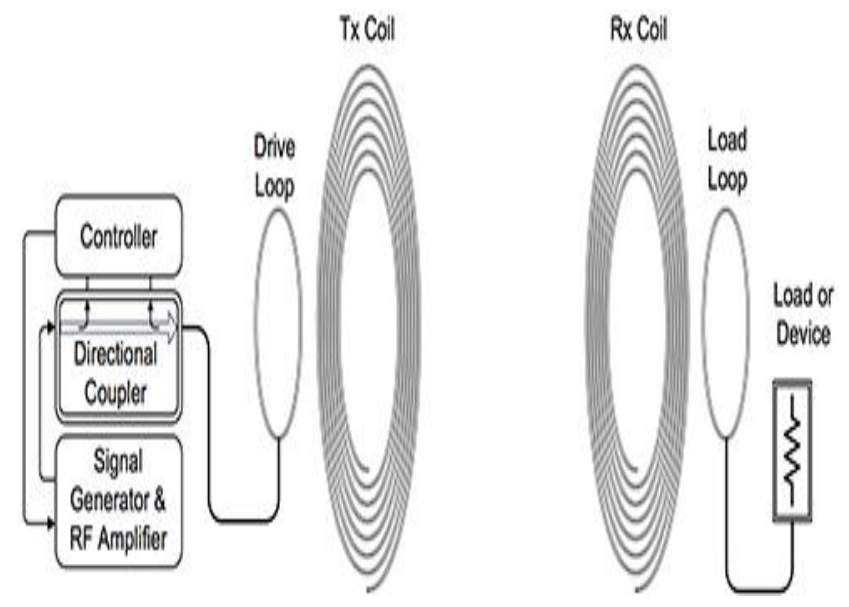


The working principle of inductive coupling is based on magnetic field, in the figure given above two coils are used one is primary coil and another one is secondary coil. When the electricity flows through the copper coil then there is some magnetic flux will occurs this magnetic flux is highly induce by the resonant circuit, the resonant circuit is used for increasing the inductance of electric power transmission. The induced magnetic field is attracted by the secondary coil which is directly connected to the load such as mobile phone charger, Television (TV), computer monitors and other lighting systems. The coil used for the transmission of power is no need to be in line of sight. During the transmission of electrical energy, it is converted into magnetic waves and then converted into electrical energy at the secondary copper coil.

\section{ADVANTAGES, DISADVANTAGES and APPLICATION}

\section{A. Advantages}

- It is suitable for most of the environment

- No need of line of sight, position independent

- Magnetic waves are not harmful to human beings

- Loss during the transmission can be removed

- One to many distribution

- The need of power cable is eliminated

- Usage of electricity will reduce

- Low cost
- Easy to maintenance

\section{B. Disadvantages}

- Efficiency of a power transfer is based on distance between transmitting and receiving coil

- Increasing the possibilities of power theft

\section{Applications}

- Automatic wireless charging point in office, home and vehicles

- Wireless TV, DVD players, Home theaters and home lighting system

- In industrial sector such as robots, packaging machine etc

\section{CONCLUSION}

The need of electricity is increased year by year to reduce the need of electricity and power loss we need a new technology. This paper proposes the inductive resonant coupling which is used to transfer the electric power from one source to many objects. Also this technology reduces the electricity waste and expenses which is needed for installing wires and also reduces the maintenance.

\section{REFERENCES}

1.The WiTricity website. [Online]. Available: http://www.witricity.com/pages/application.html

2. Tushar Supe, Aishwarya Joy, Neha Kadam, Asmita Bhagat "Study on Wireless Power Transfer Using Resonant Induction Technique" International Journal of Emerging Technology and Advanced Engineering 
3. M Maqsood and M Nauman Nasir, "Wireless electricity (Power) transmission using solar based power satellite technology”National Institute of Vacuum Science \& Technology

4. Nikola Tesla 1905 Electrical World and Engineer The Transmission of Electrical Energy Without Wires as a Means for Furthering Peace, p 21

5. Landis, Geoffrey A 2006 IEEE 4th World Conference on Photovoltaic Energy Conversion 2

6. Landis, Geoffrey A 2006 IEEE 4th World Conference on Photovoltaic Energy Conversion 21939 